\title{
Exercises in Clinical Reasoning
}

\section{A 43-Year-Old Woman with Abdominal Pain and Fever}

\author{
Craig R. Keenan, $M D^{7}$, Gurpreet Dhaliwal, $M D^{3,2}$, Mark C. Henderson, $M D^{7}$, \\ and Judith L. Bowen, $M D^{4}$
}

'Department of Internal Medicine, UC Davis Medical Center, Sacramento, CA, USA; ${ }^{2}$ Department of Medicine, University of California, San Francisco, San Francisco, CA, USA; ${ }^{3}$ San Francisco Veterans Affairs Medical Center, San Francisco, CA, USA; ${ }^{4}$ Department of Medical Informatics and Clinical Epidemiology, Oregon Health and Science University, Portland, OR, USA.

KEY WORDS: pattern recognition; analytic reasoning; problem representation; clinical diagnostic reasoning.

$\mathrm{J}$ Gen Intern Med 25(8):874-7

DOI: $10.1007 / \mathrm{s} 11606-010-1372-3$

(C) The Author(s) 2010. This article is published with open access at Springerlink.com

I $\mathrm{n}$ this series, a clinician extemporaneously discusses the diagnostic approach (regular text) to sequentially presented clinical information (bold). Additional commentary on the diagnostic reasoning process (italics) is integrated throughout the discussion.

Clinical Information: A 43-year-old Mexican woman presented to the emergency department with abdominal pain. Her illness had begun 1 week earlier with fevers to $38.9^{\circ} \mathrm{F}$ and intermittent frontal headache without photophobia or other neurological symptoms. Two days prior to presentation, she began having left upper quadrant (LUQ) abdominal pain described as sharp, unremitting and radiating to the midepigastrium, right upper quadrant and left flank. The pain did not change with eating, but she did report episodic nausea and vomiting. She denied hematemesis, dysuria or diarrhea. Her last menstrual period had begun 3 weeks earlier, and she denied recent sexual activity. One day prior she had been diagnosed with gallstones at another emergency department and treated with oral metoclopramide and hydrocodone-acetaminophen.

Clinician. Left upper quadrant pain commonly results from gastritis, colitis (splenic flexure), pancreatitis, pyelonephritis, nephrolithiasis, splenic enlargement or infarction, or left lower lobe pneumonia. Intermittent headaches frequently accompany systemic illness, so the challenge is determining if the associated fever signals an intracranial infectious process such as meningitis, encephalitis or brain abscess. The severity and duration of headache, lack of previous headache history, presence of meningeal signs and neurological deficits are common indications for imaging the central nervous system and analyzing the CSF.

Diagnostic Reasoning. The problem representation is an abstract one-sentence summary that elaborates the key features of the case. It triggers plausible diagnostic hypotheses and directs exploration of further historical

Published online May 4, 2010 elements, physical examination features and diagnostic testing. In complex cases it is often necessary to consider more than one problem representation. Here the competing versions might be: (1) a 43-year-old woman of reproductive age with a subacute febrile illness and headaches associated with LUQ abdominal pain, nausea and vomiting; or (2) a 43-year-old woman of reproductive age with acute onset of sharp, unremitting LUQ pain, nausea and vomiting. Using the first problem representation, the clinician would likely consider serious intracranial infections and mass lesions. In the second problem representation, the LUQ pain is the focal point that directs further data gathering. It is often useful to explore competing problem representations to avoid "premature closure." Premature closure is the failure to consider other plausible diagnoses after an initial working diagnosis is reached. It is one of the most common clinical reasoning errors made by clinicians. ${ }^{1}$

She had no significant past medical history. Her only medication was acetaminophen. Her family history was unremarkable. She was unemployed and denied current or prior alcohol, tobacco or drug use. She was born in Mexico and immigrated to the United States at age 23. She travels to Mexico regularly and had returned 3 weeks ago after a 2month visit. Review of systems was notable for night sweats and a 20-pound intentional weight loss over the past several months.

Although the 20-pound weight loss is described as being intentional, the rarity of that achievement and associated night sweats suggest a systemic illness such as infection, autoimmune disease or malignancy. Her travel to Mexico places her at risk for tuberculosis (gastrointestinal and CNS manifestations) and amebiasis (colitis or liver abscess). Autoimmune syndromes with gastrointestinal manifestations include systemic lupus erythematosus (SLE), polyarteritis nodosa and inflammatory bowel disease. Lymphoma would be the most likely malignancy given her age and constitutional symptoms.

Typical of complex, ambiguous cases in the early stage, the clinician appropriately considers a very broad differential diagnosis. Three categories - infection, autoimmune conditions and malignancy-provide structure for thinking through this case. The clinician uses the patient's age, gender and significant travel history to help focus the extensive differential diagnosis in each category. The problem representation is now: a 43-year-old woman with acute, unremitting LUQ pain and a subacute history of night sweats, weight loss and recent travel to Mexico.

She was in moderate distress due to abdominal pain. Her temperature was $38.1^{\circ} \mathrm{C}$, blood pressure $96 / 43 \mathrm{mmHg}$, 
heart rate 125 beats per minute and respiratory rate 16 breaths per minute. She had scleral icterus. Lung and heart exams were normal except for tachycardia. The abdomen was nondistended with normal bowel sounds, but diffusely tender, particularly in the LUQ. Liver span was $15 \mathrm{~cm}$ to percussion, and the spleen was not palpable. She had no peritoneal signs or costovertebral angle tenderness, and a pelvic examination was normal. Her neurological and skin examinations were normal.

Her appearance and vital signs suggest the systemic inflammatory response syndrome (SIRS), which requires prompt evaluation, stabilization and usually empiric antibiotic treatment. Jaundice, fever and abdominal pain prioritize acute bacterial cholangitis as an immediate concern, despite the uncertainty surrounding the nature of the subacute underlying illness. Hepatomegaly is typically explained by liver inflammation, infiltration or congestion. Massive enlargement is usually caused by an infiltrative process and is not seen in gallstone-related illnesses.

There is discordance between the presence of jaundice and hepatomegaly and the persistent LUQ pain. Plausible explanations include primary hepatic diseases with acute portal hypertension and splenic congestion; a left-sided intraabdominal infection or malignancy (e.g., diverticulitis or colon cancer) spreading to the liver via the portal circulation; or a single malignant (e.g., lymphoma), infectious (e.g., endocarditis) or infiltrative (e.g., sarcoidosis) process simultaneously affecting the liver and spleen (or another left-sided abdominal organ).

The problem representation is now: Acutely ill, possibly septic, 43-year-old woman with recent travel to Mexico, fever, diffuse abdominal pain, jaundice and hepatomegaly. The clinician recognizes the features consistent with SIRS and immediately makes recommendations for action, including empiric treatment for bacterial cholangitis. This is an example of pattern recognition. Other features of the case remain puzzling and are labeled discordant: LUQ pain with hepatomegaly and jaundice. Such discordant features require analytic reasoning, a process involving conscious hypothesis testing to direct further history, examination or diagnostic testing decisions. In all but the most straightforward clinical cases, clinicians use both pattern recognition and analytic reasoning simultaneously to establish and prioritize possible diagnoses, as illustrated here.

Initial laboratory studies were: WBC count $8,300 / \mathbf{m m} 3$ (66\% PMN, 31\% lymphocytes, 3\% monocytes), hemoglobin $12.5 \mathrm{mg} / \mathrm{dl}$, MCV $75 \mu^{3}$, platelet count $88,000 / \mathrm{mm}^{3}$, alkaline phosphatase $301 \mathrm{U} / 1$, AST $334 \mathrm{U} / 1$, ALT $133 \mathrm{U} / 1$, total bilirubin $5.1 \mathrm{mg} / \mathrm{dl}$, direct bilirubin $3.4 \mathrm{mg} / \mathrm{dl}$, albumin $2.3 \mathrm{~g} / \mathrm{dl}$ and INR 1.2. Creatinine, calcium and lipase were normal. Urinalysis showed moderate protein, 22 white blood cells per high power field and 14 red blood cells per high power field. Along with granular and hyaline casts, three white cell casts per high power field were detected.

Abdominal ultrasound showed mild hepatomegaly, mild splenomegaly, mild gallbladder wall thickening and pericholecystic fluid. There were no gallstones or biliary ductal dilatation. Chest radiograph was normal. The patient was admitted for presumed cholecystitis and treated with ceftriaxone and metronidazole.

Marked thrombocytopenia may arise from acute infection or medication toxicity, but autoimmunity, hypersplenism or an infiltrative process of the bone marrow must also be considered. The urinalysis results are compatible with pyelonephritis, but there is no history of dysuria or costovertebral angle tenderness. Other explanations for the urinary findings include glomerulonephritis or urolithiasis. Endocarditis, via immune complex deposition, could cause this active urine sediment and involve the liver.

The laboratory tests point to a combined cholestatic and hepatocellular process. The AST to ALT ratio is compatible with alcoholic hepatitis, which can present with systemic toxicity and abdominal pain; therefore, revisiting the alcohol history would be logical here. Acetaminophen levels should be checked. The sonogram results, particularly the absence of gallstones, substantially lower the likelihood of acute cholecystitis, and the left-sided abdominal pain never really supported that diagnosis. Given the biochemical evidence of direct liver involvement and unexplained left upper quadrant pain, an abdominal CT is indicated.

She has a subacute illness associated with weight loss, night sweats, SIRS, prolonged fever, LUQ pain, hepatopathy and thrombocytopenia. Leading considerations include alcoholic hepatitis, SLE with autoimmune hepatitis, lymphoma or an indolent infection. Her travel to Mexico and potential involvement of the reticuloendothelial system (liver, spleen, bone marrow) renders tuberculosis, fungal infections (e.g., histoplasmosis), endocarditis (especially if she has undiagnosed rheumatic heart disease) and brucellosis distinct possibilities. HIV testing is also indicated.

The clinician has explicitly articulated the revised problem representation in the first sentence of the paragraph above. The new data have triggered new hypotheses (e.g., alcoholic hepatitis) and eliminated earlier hypotheses (e.g., cholecystitis). The next diagnostic test (abdominal CT) is chosen to identify features that discriminate among the competing diagnoses.

CT of the abdomen showed mild hepatomegaly, mild splenomegaly and a heterogeneous spleen with multiple small hypodense lesions (Fig. 1). A hepatobiliary iminodiacetic acid (HIDA) scan was normal. The patient

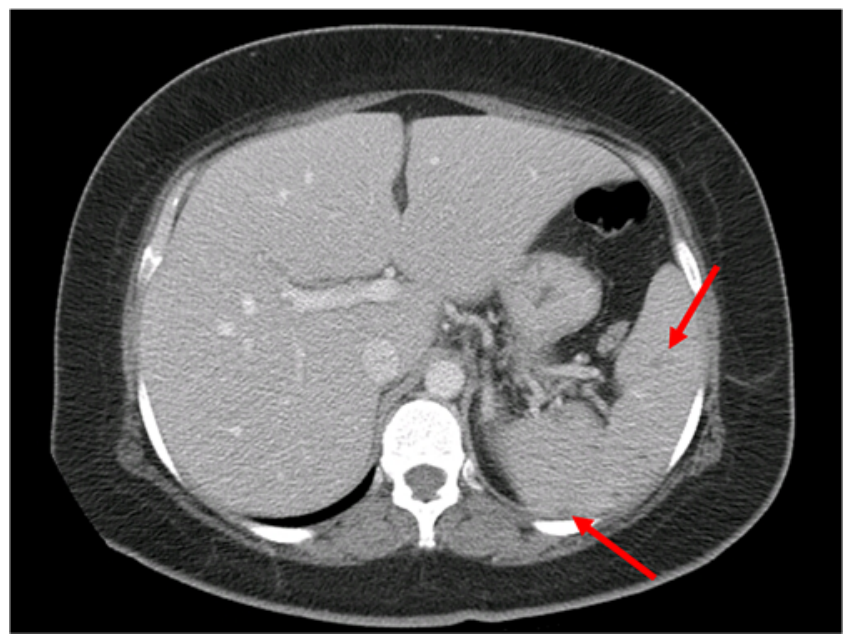

Figure 1. CT images showing hepatomegaly and small hypodense lesions in the spleen (red arrows). 
continued to have fevers. Further laboratory studies revealed negative hepatitis $A, B$ and $C$ serologies, negative HIV testing, and nonreactive RPR and PPD testing. Multiple blood and urine cultures were sterile after 2 days.

Acute cholecystitis has been excluded by a normal HIDA scan. The multiple hypodense lesions in the spleen point to an infiltrative or embolic process (causing microabscesses), and the liver enzymes implicate a similar process in the liver. The negative blood cultures substantially lower the probability of subacute bacterial endocarditis, but culturenegative endocarditis remains possible. Tuberculosis, histoplasmosis, coccioidomycosis, brucellosis, $\mathrm{Q}$ fever and bartonella infection can all present with granulomatous involvement of the liver and spleen, which would be consistent with the CT findings. Brucella is endemic to Mexico and presents with protean often nonlocalizing presentations, although she lacks the frequently noted osteoarticular manifestations (i.e., peripheral arthritis, sacroiliitis or spondylitis). Further history is warranted to assess her risk for brucella or coxiella (e.g., livestock or unpasteurized dairy product exposures), bartonella (e.g., cat, lice or flea exposures, homelessness) or tuberculosis, and to definitively exclude recent antibiotic use, a common cause of culture-negative endocarditis. A negative RPR rules out disseminated syphilis unless the patient has a prozone phenomenon (an extremely high titer of treponemes that produces a false-negative test). Coccidioidomycosis serologies and a urine histoplasma antigen would be helpful as these infections are endemic to California and Mexico, respectively. Given the significant liver pathology with negative cultures and serologic testing to date, liver biopsy would be the logical next step for diagnosis.

The clinician is now postulating diagnoses that could account for the most discriminating features in this case: multiple small hypodense splenic lesions with fever, liver test abnormalities and hepatosplenomegaly. The framework has shifted to an "infiltrative or embolic process." With the less invasive serologic tests and cultures failing to yield a specific diagnosis, the clinician recommends a liver biopsy.

Stool ova and parasite examination and stool and sputum AFB stains were negative. Antinuclear antibodies (ANA) and anti-mitochondrial antibodies were negative. Anti-smooth muscle antibody (AMSA) was 1:40, and serum protein electrophoresis was normal. Core needle liver biopsy revealed hepatitis with infiltration with chronic inflammatory cells and non-caseating granulomas. Stains were negative for fungal and acid fast elements.

The negative ANA, normal protein electrophoresis and the low titer AMSA do not support a diagnosis of autoimmune hepatitis. Granulomatous hepatitis is compatible with the aforementioned infections (TB, histoplasmosis, $\mathrm{Q}$ fever and brucellosis). It also is seen with noninfectious etiologies, such as lymphoma, sarcoidosis and primary biliary cirrhosis. Although the relevant cultures must be followed vigilantly, the negative mycobacterial and fungal stains, negative PPD and normal chest $\mathrm{X}$-ray make tuberculosis and histoplasmosis less likely. The absence of lymphadenopathy (on exam or imaging studies) makes lymphoma and sarcoidosis unlikely. A history of animal exposure or evidence of valvular heart disease would support chronic $\mathrm{Q}$ fever, which looks remark- ably similar to brucellosis. While no disease has been definitively excluded, brucellosis emerges as the leading diagnosis as it is endemic to Mexico and can account for a chronic febrile granulomatous illness involving the liver, spleen and possibly bone marrow.

The prioritization of a working differential diagnosis requires assigning probabilities to diseases that best explain the working problem representation (Table 1 ).

On the 4th hospital day, blood cultures drawn at the outside hospital emergency room returned positive for Brucella melitensis. The patient was started on doxycycline and rifampin with rapid resolution of all symptoms and laboratory abnormalities. Echocardiogram and urine histoplasmosis antigen were not ordered. Serologic studies for Coxiella burnetii were negative, and brucella serology later returned positive with IgM antibody levels of 3.53 IV (normal < $1.70 \mathrm{IV}$ ). She completed a 6-week course of antibiotics and did well. Further history revealed that when visiting her home in rural Mexico, she often milked the family cows.

\section{COMMENTARY}

In this case, the clinician employed both pattern recognition (fever, jaundice and abdominal pain triggered the diagnosis of cholangitis) and analytical reasoning to explain the jaundice and LUQ pain. Pattern recognition is a method of reasoning associated with "expertise" whereby the experienced clinician makes the correct diagnosis after asking only a few questions and performing a few physical examination maneuvers. A more advanced model of expert diagnostic reasoning embraces this ability to recognize many patterns, but also emphasizes fluidity between pattern recognition and analytical reasoning $^{3,4}$. This requires recognizing elements that do not fit the readily available pattern and further exploring those features in a focused, efficient manner. Emerging research on reasoning suggests that an approach using pattern recognition combined with careful consideration of discordant case features may improve diagnostic accuracy. ${ }^{5}$ In this complex case, multiple candidate illnesses remained plausible even after the accumulation of substantial data. Clinicians approach this problem by prioritizing the differential diagnosis in relationship to the refined problem representation. Sorting the possible diagnoses into categories of close, intermediate and limited match, and including diagnoses that pose great risk if missed, can tailor the

Table 1. Clinicians Prioritize Diagnoses by the Degree to Which Diseases Match the Working Problem Representation ${ }^{2}$

Problem representation: Young woman, Mexico, subacute febrile illness, thrombocytopenia, multiple splenic lesions and granulomatous hepatitis

\begin{tabular}{ll}
\hline Diagnostic tier & Diagnostic considerations \\
\hline $\begin{array}{l}\text { I (close match) } \\
\text { Ib (potentially fatal in } 24 \mathrm{~h} ; \\
\text { variable match) }\end{array}$ & $\begin{array}{l}\text { Brucellosis } \\
\text { Cholangitis (acute presentation) } \\
\text { II (intermediate match) }\end{array}$ \\
$\begin{array}{l}\text { Lymphoma, Q fever, tuberculosis, } \\
\text { histoplasmosis } \\
\text { III (limited match) }\end{array}$ & $\begin{array}{c}\text { Sarcoidosis, SLE, alcoholic hepatitis, } \\
\text { cholecystitis, autoimmune hepatitis }\end{array}$ \\
&
\end{tabular}


diagnostic workup for greater efficiency and cost-effectiveness (Table 1).

\section{CLINICAL TEACHING POINTS}

1. Human brucellosis is a very common illness worldwide. Four brucella species-melitensis, suis, abortus and caniscause the majority of human illness, with $B$. melitensis being the most common. ${ }^{6}$

2. Brucellosis is generally transmitted by direct contact with infected animals (cattle, sheep, goats or pigs) or by consuming unpasteurized dairy products. The incubation period is days to months.

3. Though brucellosis can involve any organ system, it usually presents with nonspecific symptoms, including undulating fevers, night sweats, malaise, weight loss and arthralgias. It can also present with focal brucellosis syndromes, including meningitis, arthritis, endocarditis or epididymoorchitis.

4. Granulomatous hepatitis is caused by infections (e.g., tuberculosis, histoplasmosis, coccidioidomycosis, $\mathrm{Q}$ fever, brucellosis, syphilis, cryptococcosis, leprosy), drugs, malignancy (e.g., lymphomas, renal cell carcinoma) or autoimmune diseases (e.g., sarcoidosis, polymyalgia rheumatica, primary biliary cirrhosis). Up to 20 percent of cases are idiopathic. $^{7}$

5. The social history is often a key discriminating feature in febrile illnesses, particularly for patients who travel outside of industrialized areas. They are often exposed to infections that are not routinely considered.
Acknowledgement: The authors thank Hien Nguyen, MD, at the UC Davis Medical Center for his invaluable review of the manuscript.

Open Access: This article is distributed under the terms of the Creative Commons Attribution Noncommercial License which permits any noncommercial use, distribution, and reproduction in any medium, provided the original author(s) and source are credited.

Corresponding Author: Craig R. Keenan, MD, Department of Internal Medicine, UC Davis Medical Center, 4150 V Street, Suite 2400, Sacramento, CA 95817, USA (e-mail: craig.keenan@ucdmc. ucdavis.edu).

\section{REFERENCES}

1. Graber ML, Franklin N, Gordon R. Diagnostic error in internal medicine. Arch Intern Med. 2005;165:1493-9.

2. Lucey C. APDIM National Meeting. Philadelphia; 2000.

3. Eva KW, Regehr G. Knowing when to look it up: a new conception of selfassessment ability. Acad Med. 2007;82(10 Suppl):S81-4.

4. Moulton CA, Regehr G, Mylopoulos M, MacRae HM. Slowing down when you should: a new model of expert judgment. Acad Med. 2007;82(10 Suppl):S109-16.

5. Hatala EKW, RM LeBlanc VR, Brooks LR. Teaching from the clinical reasoning literature: combined reasoning strategies help novice diagnosticians overcome misleading information. Med Educ. 2007;41:11528.

6. Pappas G, Akritidis N, Bosilkovski M, Tsianos E. Brucellosis. N Engl J Med. 2005;352:2325-36.

7. Matheus T, Munoz S. Granulomatous liver disease and cholestasis. Clin Liver Dis. 2004;8:229-46. 